\title{
Sensitivity In X-Ray Grating Interferometry On Compact Systems
}

\author{
Thomas Thuering ${ }^{*, \dagger}$, Peter Modregger ${ }^{*, * *}$, Stefan Hämmerle ${ }^{\sharp}$, Stephan \\ Weiss ${ }^{\ddagger}$, Joachim Nüesch ${ }^{\ddagger}$ and Marco Stampanoni ${ }^{*, \dagger}$ \\ ${ }^{*}$ Swiss Light Source, Paul Scherrer Institut, Villigen PSI, Switzerland \\ ${ }^{\dagger}$ Institute for Biomedical Engineering, Swiss Federal Institute of Technology, Zurich, Switzerland \\ ${ }^{* *}$ School of Biology and Medicine, University of Lausanne, Lausanne, Switzerland \\ ${ }^{\ddagger}$ SCANCO Medical AG, Bruettisellen, Switzerland
}

\begin{abstract}
The optimization of compact X-ray grating interferometry systems is crucial for the progress of this technique in industrial devices. Here, an analytical formulation for the sensitivity of the phase contrast image acquisition is derived using previous results from noise analyses. Furthermore, experimental measurements of the sensitivity for different configurations are compared, providing further insight into the dependence on polychromatic radiation. Finally, strategies for the geometrical optimization are given.
\end{abstract}

Keywords: X-ray phase contrast imaging, grating interferometry, compact micro CT PACS: $07.85 . \mathrm{Tt}$

\section{INTRODUCTION}

X-ray grating interferometry represents a multi-modal imaging technique for the acquisition of absorption, differential phase [1] and dark field [2] radiographs and tomograms. The grating interferometer (GI) requires high spatial beam coherence and thus it is most prominently applied on synchrotron sources. Using microfocus X-ray tubes [3] or a source grating [4], GI has also been applied on conventional X-ray tubes. In phase contrast mode, a radiograph (phase projection) is indirectly acquired by measuring the refraction angle (sub-pixel refraction) caused by the object. Refraction angle and projected phase profile are related by a first derivative. The sensitivity of the GI can therefore be characterized by the minimum detectable refraction angle [5].

The determination of the relation between GI system parameters and the sensitivity is crucial for the optimization of a setup. Virtually all GI system parameters affect the sensitivity. They can roughly be divided into X-ray source related parameters (e.g., coherence, flux), detector related parameters (e.g., detection efficiency, pixel size), geometry related parameters (e.g., grating positions, grating periods, sample position) and measurement parameters (e.g. exposure time, number of phase steps). For instance, the sensitivity of grating interferometry on synchrotron sources is typically higher than for tube based setups, which is mainly due to the narrow energy bandwidth (temporal coherence) and the higher photon flux at synchrotrons.

In this contribution, an analytical formulation of the sensitivity as a function of such parameters, mainly geometry, source and detector related, is derived. Based on that, the optimum geometry of the GI which maximizes the sensitivity is determined.

International Workshop on X-ray and Neutron Phase Imaging with Gratings

AIP Conf. Proc. 1466, 293-298 (2012); doi: 10.1063/1.4742307

(C) 2012 American Institute of Physics 978-0-7354-1072-5/\$30.00 
Furthermore, experimental measurements of the sensitivity on extremely compact setup lengths using bendable gratings [6] are demonstrated. Special attention is given to the sensitivity as a function of the total setup length, which is a crucial issue for the design of compact GI systems.

\section{THEORY}

\section{Setup geometry}

Figure 1 schematically shows the geometry of a compact GI setup with bendable gratings. The gratings are labeled by G0 (source grating), G1 (beam splitter grating) and G2 (analyzer grating), whereas the grating periods are given by $p_{0}, p_{1}$ and $p_{2}$. Bendable gratings allow the design of extremely compact systems without a decrease in the field of view [6]. Especially for the source grating, which is preferably positioned as close as possible to the source, the radius is very short, requiring a flexible substrate for bending. The parameter $m$ is the fractional Talbot order $(m \in\{1,3,5, \ldots\}), \lambda$ is the wavelength and $\eta$ indicates whether the beam splitter grating is a $\pi / 2$-shifting $(\eta=1)$ or a $\pi$-shifting $(\eta=2)$ phase grating. The following formulae give the basic relations of the geometry:

$$
\begin{gathered}
s=d+l, \\
d=d_{m}=\frac{l \cdot \frac{m}{\eta^{2}} d_{T}}{l-\frac{m}{\eta^{2}} d_{T}}, \\
d_{T}=\frac{p_{1}^{2}}{2 \lambda}, \\
p_{2}=\frac{p_{1}}{\eta} \frac{s}{l}, \\
p_{0}=\frac{l}{d} p_{2} . \\
M_{\mathrm{S}} \approx \frac{s}{l_{s}}
\end{gathered}
$$

Eq. (6) refers to the geomeric magnification of the sample in the divergent beam. In Eq. (6) and in the following, the setup length is approximated by $s$, although the total setup length is in reality slightly longer due to spacings between source and G0 and between G2 and the detector.

\section{Sensitivity}

The sensitivity of the GI is (inversely) related to the smallest detectable refraction angle [5], $\alpha_{\min }$, which is proportional to the smallest detectable interference fringe shift, $\varphi_{\min }$, given by

$$
\alpha_{\min }=\frac{p_{2}}{2 \pi d} \frac{l}{l_{s}} \varphi_{\min }
$$




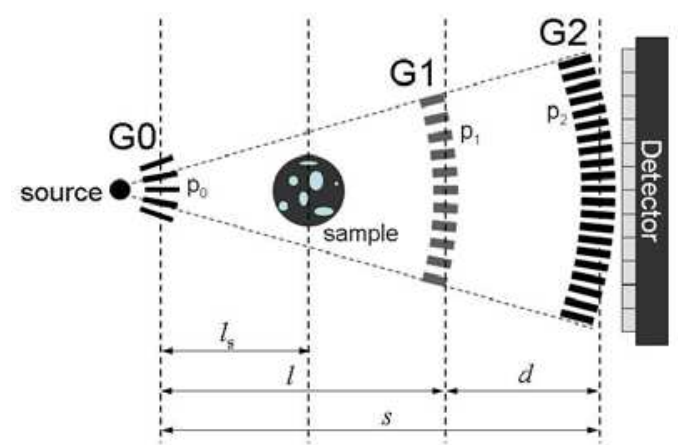

FIGURE 1. GI setup for a compact (cone-beam) geometry. The source grating G0 generates an array of coherent (mutually incoherent) sources, G1 generates an interference pattern at the position of G2 and $\mathrm{G} 2$ analyzes the interference fringes.

where $p_{2} /(2 \pi d)$ accounts for the propagation distance of the beam downstream of the beam splitter grating and $l / l_{s}$ is the sensitivity reduction related to the sample position $\left(l_{s} \leq l\right)$ [3]. The detectability of $\alpha$ depends on the standard deviation of this signal, and thus

$$
\alpha_{\min } \equiv \sigma_{\alpha}=\frac{p_{2}}{2 \pi d} \frac{l}{l_{s}} \sigma_{\varphi}
$$

Using Eq. (1) and (4), the inter-grating distance $d$ can be expressed as

$$
d=s \frac{\eta p_{2}-p_{1}}{\eta p_{2}} .
$$

The sample related sensitivity factor $l / l_{S}$ can be expressed using the magnification $M_{\mathrm{S}}$, yielding

$$
\frac{l}{l_{s}}=M_{\mathrm{S}}-\frac{d}{l_{s}}=M_{\mathrm{S}}\left(1-\frac{\eta p_{2}-p_{1}}{\eta p_{2}}\right)
$$

Furthermore, an analytical formula for $\sigma_{\varphi}$ has been derived by Engel et al. [7] and is given by

$$
\sigma_{\varphi}=\frac{\sqrt{2}}{V \sqrt{N}}
$$

where $V$ is the normalized magnitude of the first Fourier component of the phase stepping curve (visibility) and $N$ is the expectation value for the number of photons. Since $V$ and $N$ can vary across the field of view due to source/detector inhomogeneities or grating imperfections, the sensitivity $\alpha_{\min }$ becomes pixel dependent. Therefore in practice, the result of a sensitivity measurement is typically a sensitivity map.

Using Eqs. (9)-(11), Eq. (8) can be reformulated to

$$
\alpha_{\min }=\frac{1}{\sqrt{2} \pi} \frac{1}{s} M_{\mathrm{s}} \frac{p_{1} p_{2}}{\eta p_{2}-p_{1}} \frac{1}{V \sqrt{N}} .
$$

For X-ray tube based setups, the number of photons $N$ is not only source and detector dependent, it is also affected by the setup length. A setup length independent quantity is 
the beam intensity per unit solid angle and unit time $I_{0}$ [8]. Using $I_{0}, N$ can be expressed as

$$
N=I_{0} D \frac{a^{2}}{s^{2}} n_{\mathrm{ps}} t
$$

where $a$ is the pixel size (assuming quadratic pixels), $D$ the detector efficiency ( $D \in$ $[0,1]), n_{\mathrm{ps}}$ the number of phase steps and $t$ the exposure time per phase step. Again, the setup length was approximated by $s$. Putting this equation into Eq. (12) finally yields for the sensitivity

$$
\alpha_{\min }=\alpha_{\min }(t)=\frac{1}{\sqrt{2} \pi} M_{\mathrm{s}} \frac{p_{1} p_{2}}{\eta p_{2}-p_{1}} \frac{1}{\sqrt{n_{\mathrm{ps}} t}} \frac{1}{V \sqrt{I_{0} D} a}=\frac{1}{\sqrt{2} \pi} \gamma_{\mathrm{obj}} \gamma_{\mathrm{GI}} \gamma_{\mathrm{m}} \gamma_{\mathrm{sd}}
$$

This equation shows the separable factors which contribute to the sensitivity, including an object position dependent factor $\left(\gamma_{\mathrm{obj}}=M_{\mathrm{S}}\right)$, a GI related factor $\left(\gamma_{\mathrm{GI}}=p_{1} p_{2} /\left(\eta p_{2}-\right.\right.$ $\left.\left.p_{1}\right)=p_{0}\right)$, a factor related to measurement parameters $\left(\gamma_{\mathrm{m}}=1 / \sqrt{n_{\mathrm{ps}} t}\right.$ and a factor considering the source and detector properties $\left(\gamma_{\mathrm{sd}}=1 /\left(V \sqrt{I_{0} D} a\right)\right)$.

If we incorporate the fact that the achievable grating periods (or aspect ratios, especially of G0 and G2) are limited by the fabrication process, $\gamma_{\mathrm{GI}}$ is minimized for $p_{1}=$ $\eta p_{2} / 2$ (implying also that $p_{0}=p_{2}$ ), which represents the symmetric case $(d=l=s / 2$ ). An optimal setup design should therefore always be symmetric.

In the symmetric case, where the GI related factor simplifies to $\gamma_{\mathrm{GI}}=p_{0}=p_{2}, \alpha_{\min }$ is minimized for the smallest achievable grating periods.

The choice of the beam splitter grating parameter $\eta$ has no effect to the sensitivity. However, using $\eta=2$ ( $\pi$-shifting) in a symmetric configuration allows to fabricate all of the three gratings with the same period $\left(p_{0}=p_{1}=p_{2}\right)$, while this does not apply for $\eta=1\left(\pi / 2\right.$-shifting, $\left.p_{0}=2 p_{1}=p_{2}\right)$.

The dependence on the measurement parameters given by $\gamma_{m}$ shows, that the sensitivity only depends on the total exposure time $n_{\mathrm{ps}} t$, meaning that the number of phase steps is not a crucial parameter as long as the total exposure time is maintained.

It is important to note that the dependency of $\alpha_{\min }$ on $s$ canceled out in Eq. (14). This means that for a given grating set $\left(p_{0}, p_{1}\right.$ and $\left.p_{2}\right)$, the sensitivity is invariant to any setup length changes, although such changes are of course limited to the allowed fractional Talbot orders $(m=1,3,5, \ldots)$. Essentially, the gain in sensitivity due to the increased number of photons for a shorter setup is equal to the sensitivity loss due to the shorter inter-grating distance. However, it will be shown in the next section that this result is not entirely true in practice.

\section{EXPERIMENTAL SENSITIVITY ANALYSIS}

Although Eq. (14) is invariant to the wavelength and thus valid for arbitrary photon energies, it does not account for any effect by the spectral bandwidth. The spectral bandwidth predominantly affects the visibility and consequently has an effect to the sensitivity. Measurements of the sensitivity for two different setup lengths (or different fractional Talbot orders), which are both symmetric (i.e. geometrically optimized), have been performed. The detailed setup parameters are listed in Table 1. The X-ray tube 
TABLE 1. Setup parameters for the sensitivity measurements

\begin{tabular}{lll}
\hline Parameter & Setup 1 & Setup 2 \\
\hline$p_{0}=p_{1}=p_{2}$ & $2.4 \mu \mathrm{m}$ & $2.4 \mu \mathrm{m}$ \\
$s$ & $195 \mathrm{~mm}$ & $325 \mathrm{~mm}$ \\
$m$ & 3 & 5 \\
$V$ & $6 \%$ & $5 \%$ \\
$\eta$ & 2 & 2 \\
$a$ & $48 \mu \mathrm{m}$ & $48 \mu \mathrm{m}$ \\
$E_{\mathrm{d}}$ & $28 \mathrm{keV}$ & $28 \mathrm{keV}$ \\
$n_{\mathrm{ps}}$ & 16 & 16 \\
$t$ & $8 \mathrm{~s}$ & $8 \mathrm{~s}$ \\
\hline$\alpha_{\text {min }}$ & $270 \mathrm{nrad}$ & $300 \mathrm{nrad}$ \\
\hline
\end{tabular}

voltage and current was $50 \mathrm{kV}$ and $200 \mu \mathrm{A}$, respectively. The measurement included the acquisition of 100 phase stepping scans (16 steps each), from which the standard deviation of the phase, $\sigma_{\varphi}$, was calculated in each pixel. The sensitivity map was then determined using Eq. (7). The last line of Table 1 displays the mean values of $\alpha_{\min }$ over the field of view. As a matter of fact, the mean sensitivity of the more compact setup 1 is better than the one for setup 2. This result cannot be explained by Eq. (14), where the sensitivity is independent of the setup length for constant grating parameters. The explanation lies in the reduced visibility $V$ for setup 2, which is a consequence of the polychromatic beam. The reduction of the visibility due to the spectral bandwidth is more pronounced at higher Talbot orders (larger inter-grating distances) [9]. This result shows, that the sensitivity is not completely invariant to the setup length, at least for the polychromatic case. More importantly, it shows that the sensitivity can be maximized by minimizing the setup length.

\section{CONCLUSION}

An analytical derivation for the minimum detectable refractive angle on tube based, compact GI systems, has been presented. It illustrated the dependence of this sensitivity measure on separable factors including sample magnification, GI parameters, measurement parameters and source and detector properties, respectively. For a given total setup length, the sensitivity is optimized if the GI geometry is symmetric. Furthermore, it was shown that for given grating parameters, the sensitivity is independent on the setup length, if the beam is monochromatic. For the polychromatic case, experimental measurements showed that the sensitivity is optimized for a minimum total setup length.

\section{ACKNOWLEDGEMENTS}

We acknowledge Gordan Mikuljan from Paul Scherrer Institute, Switzerland, for his great support on several hardware design and manufacturing issues for this work. 


\section{REFERENCES}

1. C. David, B. Nöhammer, H. Solak, and E. Ziegler, Applied Physics Letters 81, 3287-3289 (2002).

2. F. Pfeiffer, M. Bech, O. Bunk, P. Kraft, E. Eikenberry, C. Brönnimann, C. Grünzweig, and C. David, Nature Materials 7, 134-137 (2008).

3. M. Engelhardt, J. Baumann, M. Schuster, C. Kottler, F. Pfeiffer, O. Bunk, and C. David, Applied Physics Letters 90, 224101 (2007).

4. F. Pfeiffer, T. Weitkamp, O. Bunk, and C. David, Nature Physics 2, 258-261 (2006).

5. P. Modregger, B. Pinzer, T. Thüring, S. Rutishauser, C. David, and M. Stampanoni, Optics Express 19, 18324-18338 (2011).

6. T. Thüring, P. Modregger, T. Grund, J. Kenntner, C. David, and M. Stampanoni, Applied Physics Letters 99, 041111 (2011).

7. K. Engel, D. Geller, T. Köhler, G. Martens, S. Schusser, G. Vogtmeier, and E. Rössl, Nuclear Instruments and Methods in Physics Research Section A 648, 202-207 (2010).

8. W. Yashiro, Y. Takeda, and A. Momose, Journal of the Optical Society of America. A 25, 2025-2039 (2008).

9. T. Weitkamp, A. Diaz, C. David, F. Pfeiffer, M. Stampanoni, P. Cloetens, and E. Ziegler, Optics Express 12, 6296-6304 (2005). 